\title{
The association between the quick sequential organ failure assessment score prior to emergency ICU admission and outcomes in adults with suspected infection
}

\author{
S Pillay, (D) T Kisten, (iD) HM Cassimjee (iD) \\ Department of Anaesthesiology and Critical Care, University of KwaZulu-Natal, South Africa \\ Corresponding author, email: sunthurie.pillay@gmail.com
}

Background: Sepsis and septic shock are leading causes of mortality world-wide. ${ }^{1}$ In patients outside the intensive care unit (ICU) a rising qSOFA (quick Sequential Organ Failure Assessment) score correlates with mortality risk. ${ }^{2,3}$ We sought to investigate if the duration of a qSOFA score $\geq 2$ prior to ICU admission further affects outcomes, namely: ICU mortality, in-hospital mortality and length of ICU stay.

Method: A retrospective chart review was performed using the electronic ICU database at a quaternary level hospital in Durban, KwaZulu Natal, examining entries from 1 January 2008 to 31 December 2017. The review included 235 emergency in-hospital adult admissions with suspected infection, of which 144 had a qSOFA score $\geq 2$ prior to ICU admission.

Results: There was no significant association between the duration of a qSOFA score $\geq 2$ prior to ICU admission and ICU mortality $(p=0.975)$, in-hospital mortality $(p=0.918)$ and length of ICU stay until demise $(p=0.848)$ or discharge $(p=0.624)$. The qSOFA score was significantly associated with ICU mortality with scores of $0,1,2$ and 3 resulting in ICU mortality rates of $0 \%, 22.5 \%, 53.7 \%$ and $84.6 \%$ respectively $(p<0.001)$.

Conclusion: The duration of a qSOFA score $\geq 2$ prior to emergency ICU admission was not significantly associated with ICU mortality, in-hospital mortality or length of ICU stay in adults with suspected infection.

Keywords: sepsis, qSOFA

\section{Introduction}

Sepsis and septic shock are disease states that remain elusive to any one diagnostic test, with the definitions themselves evolving with time. Despite these disparities in previous definitions, they are still noted as leading causes of mortality globally with as many as 1 in 4 of those afflicted dying. ${ }^{1}$ In 2016, the Third International Consensus Definitions for Sepsis and Septic Shock Task Force published updated working definitions and suggested a new risk assessment tool, the qSOFA score. ${ }^{4}$ Sepsis is defined as "lifethreatening organ dysfunction caused by a dysregulated host response to infection" and septic shock was defined as "a subset of sepsis in which particularly profound circulatory, cellular, and metabolic abnormalities are associated with a greater risk of mortality than sepsis alone". ${ }^{\prime}$

qSOFA was designed as a bedside score outside of the ICU environment, to quickly identify patients with suspected infection who are at an increased risk of poor outcomes - namely increased in-hospital mortality and prolonged ICU stays ( $\geq 3$ days). ${ }^{4}$ It was developed to identify patients who would benefit from further evaluation for the presence of possible sepsis or indeed infection (if it was not considered previously) prompt more frequent observation and initiation or escalation of therapy as appropriate. ${ }^{2}$ The components of the qSOFA score are the systolic blood pressure (SBP), the respiratory rate (RR) and the Glascow Coma Scale (GCS). A score of 1 is allocated if the $\mathrm{SBP} \leq 100 \mathrm{mmHg}$ or the RR $\geq 22$ breaths per minute or the GCS $<15$. A qSOFA score of 2 or more was suggested as the prompt for clinicians to investigate further and initiate or escalate therapy as needed. ${ }^{4}$ Despite an absence of laboratory criteria, qSOFA was noted to have similar, and in some studies, better predictive value than the full SOFA score outside of the ICU., ${ }^{4,5}$ The mortality rate for patients with a qSOFA score of 2 or more was $24 \%$ in the seminal study by Freund et al., ${ }^{3}$ but this accounted for $70 \%$ of the deaths due to sepsis. These findings prompted other researchers to evaluate the qSOFA score, with conflicting results.,

The qSOFA score was derived from databases in the United States of America (USA), and analysed in both American and international (European) settings, ${ }^{2}$ however there were still calls for external validation from low- and middle-income countries. ${ }^{8}$ Two studies based in the African context, namely Malawi and Gabon, showed a promising prognostic value of the qSOFA score in resource limited settings., ${ }^{9,10}$ Larger scale studies have since been performed, which confirmed the utility of qSOFA to predict mortality in the setting of sepsis, ${ }^{8}$ but it is still uncertain whether this translates to earlier detection of sepsis and improved outcomes. ${ }^{11}$

Even in a high income country, such as the USA, Lundberg et al. ${ }^{12}$ noted that patients who had developed septic shock as inpatients had a higher mortality rate than those admitted directly to ICU. Patients in general wards also had significant delays in referral to ICU and institution of therapeutic measures when compared to mean time of commencing therapy in ICU. Shorr et al. ${ }^{13}$ similarly demonstrated the association between a delay in ICU admission of septic patients and an increase in mortality, 
length of hospital stay and incurred costs. Gonçalves-Pereira et al. ${ }^{14}$ in the INFAUCI trial, however, found no significant difference in mortality rates between direct ICU admission and prior admission to a general ward, and emphasised early appropriate antibiotic therapy as a key modifiable factor in reducing mortality regardless of where it was initiated. The authors also highlighted the important non-modifiable factors that contribute towards mortality such as age, functional status and comorbidities. ${ }^{14}$

This study was undertaken to determine whether the qSOFA criteria was able to prognosticate outcomes in this study population. In addition, the association of the duration to ICU admission from a qSOFA $\geq 2$ with outcomes was evaluated in this resource limited setting.

\section{Method}

\section{Study design}

This was an observational retrospective study.

\section{Study population}

The study population included all adult in-hospital emergency admissions (age 18 years old and above) with a confirmed or suspected infection-related diagnosis on admission to ICU2, a multidisciplinary unit at Inkosi Albert Luthuli Central Hospital between the period of 1 January 2008 and 31 December 2017 ( $n=235$ ). Of the 235 eligible patients admitted to the ICU within the study period, 144 had a qSOFA score $\geq 2$ prior to ICU admission.

The exclusion criteria were: elective admissions, no suspicion of infection on admission, patients below 18 years of age, repeat ICU admissions from the same hospital episode, direct emergency ICU admissions from other hospitals, and patients transferred to another ICU facility whose eventual outcome was unknown.

\section{Data extraction}

Using the ICU2 database, data was extracted over the period of 1 January 2008 to 31 December 2017. Adult in-hospital emergency admissions were identified, and on review of admission diagnosis they were included if an infection-related diagnosis or suspected infection was noted. Patients' electronic medical records were reviewed to extract the necessary information to populate data collection tool fields and exclusions were made as necessary. One point was allocated for the presence of each qSOFA parameter and the qSOFA score calculated by their sum. The time of a qSOFA score $\geq 2$ was taken as the point in time prior to ICU admission at which a score of 2 or greater was reached. It was not the time of maximal qSOFA prior to admission. Time from a qSOFA score $\geq 2$ to ICU admission was calculated. Anonymity was maintained as study numbers were allocated to each member of the study population.

\section{Statistics}

\section{Statistical methodology}

IBM SPSS was used to analyse the data. A $p$-value $<0.05$ was considered as statistically significant. Associations between categorical variables were tested using Pearson's chi square tests or Fisher's exact tests as appropriate. Non-parametric KruskalWallis tests were used to compare non-normally distributed outcomes between independent groups. Kaplan-Meier survival analysis was used to analyse time to event data and log rank tests were used to compare survival between groups of patients. Logistic regression was used to analyse the association between various durations of a qSOFA score $\geq 2$ prior to ICU admission and outcomes. Primary outcomes were those associations in relation to duration of a qSOFA score $\geq 2$ namely ICU mortality rate, in-hospital mortality rate (ICU mortality plus mortality after ICU discharge), and length of ICU stay. Secondary outcomes were associations related to the qSOFA score and comorbidities.

\section{Demographics}

Table I: Demographics and baseline comorbidities of the study population

\begin{tabular}{|c|c|}
\hline Variable & $n=235$ \\
\hline \multicolumn{2}{|l|}{ Age (years) } \\
\hline Mean (standard deviation) & $46.7(+/-17.8)$ \\
\hline \multicolumn{2}{|l|}{ Sex } \\
\hline Male & $107 *(45.5 \%)$ \\
\hline Female & $128(54.5 \%)$ \\
\hline \multicolumn{2}{|l|}{ Comorbidities } \\
\hline None & $31(13.2 \%)$ \\
\hline Present & $204(86.8 \%)$ \\
\hline \multicolumn{2}{|l|}{ Number of comorbidities } \\
\hline 1 & $87(37 \%)$ \\
\hline 2 & $69(29.4 \%)$ \\
\hline 3 & $34(14.5 \%)$ \\
\hline 4 & $13(5.5 \%)$ \\
\hline 5 & $1(0.4 \%)$ \\
\hline Hypertension & $80(34 \%)$ \\
\hline Diabetes mellitus & $46(19.6 \%)$ \\
\hline Congestive cardiac failure & $10(4.3 \%)$ \\
\hline Chronic kidney disease & $32(13.6 \%)$ \\
\hline Chronic obstructive pulmonary disease & $11(4.7 \%)$ \\
\hline Hepatic insufficiency & $6(2.6 \%)$ \\
\hline Immunocompromised & $95(40.4 \%)$ \\
\hline Other & $104(44.3 \%)$ \\
\hline
\end{tabular}

*in brackets as a percentage of total population

The age range of the study population included patients from 18 to 87 years old with a mean age of 46.7 years. The gender distribution showed female patients accounting for $54.5 \%$ of the study population and males $45.5 \%$. Most patients had several comorbidities with hypertension being the most common. A large proportion of patients had comorbidities which fell into the grouped categories of other and immunocompromised. Other comorbidities included baseline comorbidities that did not fall into the categories stipulated above, examples include 
asthma, inflammatory bowel disease, thyroid disease, epilepsy, valvular heart disease and arrhythmias among others.

\section{Study population}

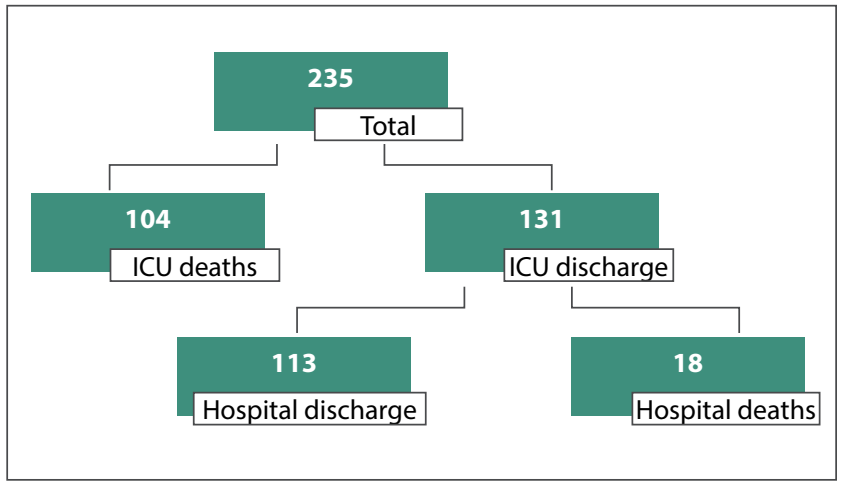

Figure 1: Total study population

235 patients met the inclusion criteria for the study. Of the 235 admissions to ICU, 104 demised. The ICU mortality rate was $44.3 \%$ (104/235). Of the 131 patients who were discharged, a further 18 died in hospital and 113 were discharged home or to their base hospital. The total in-hospital mortality rate was $51.9 \%$ (122/235).

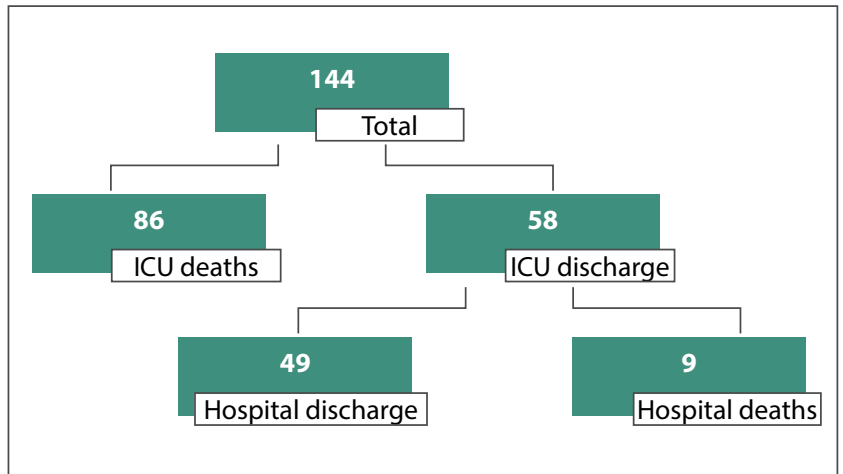

Figure 2: Population subset with a qSOFA score $\geq 2$ prior to ICU admission

Of the 235 admissions, 144 had a qSOFA score $\geq 2$ prior to admission. Of these patients 86 died in ICU and a further 9 died in hospital after ICU discharge. Among patients with a qSOFA $\geq 2$ score prior to ICU admission, the ICU mortality rate was $59.7 \%$ $(86 / 144)$ and total in-hospital mortality rate was $66 \%$ (95/144).

\section{Results}

\section{qSOFA scores prior to ICU admission}

Table II: qSOFA scores of study population prior to ICU admission

\begin{tabular}{cc}
\hline qSOFA score & Total number of patients $(\boldsymbol{n}=\mathbf{2 3 5})$ \\
\hline 0 & $11(4.7 \%)^{*}$ \\
1 & $80(34 \%)$ \\
2 & $131(55.7 \%)$ \\
3 & $13(5.5 \%)$ \\
\hline
\end{tabular}

*in brackets as a percentage of total study population
As displayed in Table II above, there were 91 patients who did not achieve a qSOFA score $\geq 2$ prior to ICU admission. These patients were predominantly direct admissions from theatre who had deteriorated intraoperatively.

\begin{tabular}{cc} 
Table III: Duration of a qSOFA score $\geq 2$ & prior to ICU admission \\
\hline Duration of qSOFA score $\geq \mathbf{2}$ & Total subset $(\boldsymbol{n}=\mathbf{1 4 4})$ \\
\hline$<48$ hours & $79(54.9 \%)^{*}$ \\
$\geq 48$ hours to $<96$ hours & $21(14.6 \%)$ \\
$\geq 96$ hours & $44(30.6 \%)$ \\
\hline
\end{tabular}

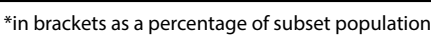

From the subset population who had qSOFA scores of 2 and 3 , the median length of time from meeting a qSOFA score $\geq 2$ to ICU admission was 38 hours (total range 0 to 2555 hours). This was a very wide range, so upon further review it was decided to separate the duration of a qSOFA score $\geq 2$ into arbitrary time bands: duration of a qSOFA score $\geq 2$ : less than 48 hours, 48 hours and greater but less than 96 hours, and 96 hours and greater.

\section{Primary outcomes}

\section{Duration of a qSOFA score $\geq 2$ prior to ICU admission and outcomes}

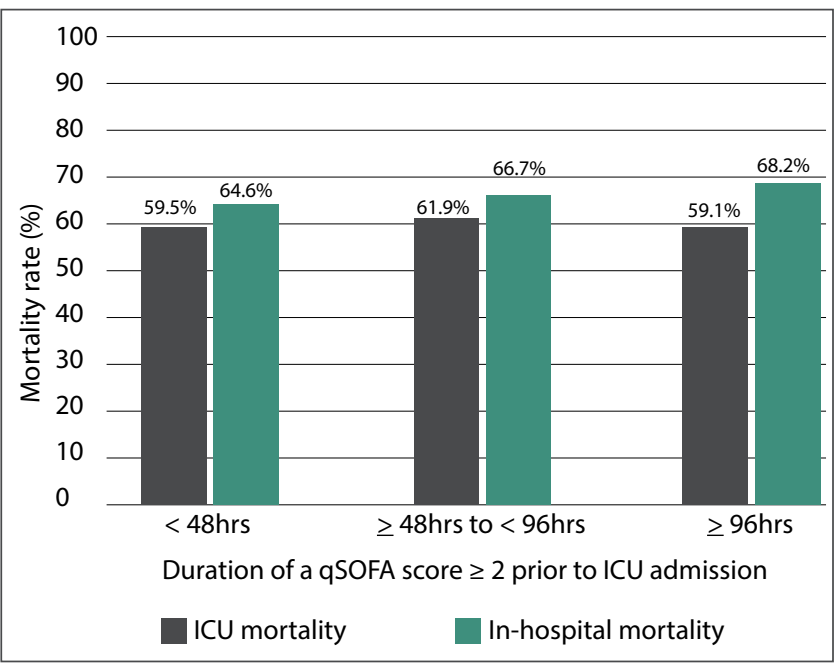

Graph 1: ICU and in-hospital mortality rate per time band of a qSOFA score $\geq 2$

\section{ICU mortality}

A logistic regression was performed. No significant association was found between the duration of a qSOFA score $\geq 2$ prior to ICU admission and ICU mortality $(p=0.975)$. The odds ratio (OR) comparing the groups of $<48$ hours and $\geq 48$ hours to $<96$ hours was 1.106 [95\% confidence interval $(\mathrm{Cl}) 0.412-2.974]$ ( $p=0.841)$, and comparing $<48$ hours and $\geq 96$ hours was $0.983[95 \% \mathrm{Cl}$ 0.464-2.083] $(p=0.965)$.

\section{In-hospital mortality}

A logistic regression showed no significant association between the duration of a qSOFA score $\geq 2$ prior to ICU admission and in-hospital mortality ( $p=0.918$ ). The OR comparing the groups of $<48$ hours and $\geq 48$ hours to $<96$ hours was $1.098[95 \% \mathrm{Cl}$ 
0.397-3.038] ( $p=0.857)$, and comparing $<48$ hours and $\geq 96$ hours was 1.176 [95\% Cl 0.537-2.577] $(p=0.685)$.

\section{Length of ICU stay}

The median length of ICU stay until demise was 90 hours (total range 0-1 613 hours) and until discharge was 94.5 hours (total range 12-1 980 hours). There was no correlation between duration of a qSOFA score $\geq 2$ prior to ICU admission and length of ICU stay until demise ( $p=0.848)$ or discharge $(p=0.624)$.

\section{Secondary outcomes}

\section{qSOFA score and outcomes}

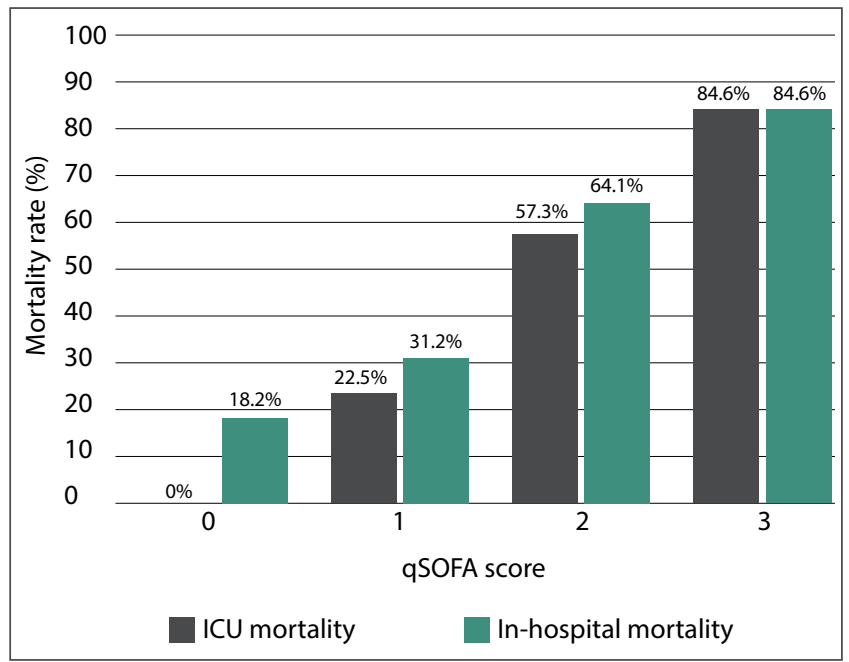

Graph 2: ICU and in-hospital mortality rate per qSOFA score

There was a linear increase in probability of ICU mortality as qSOFA score increased. The association was significant $(p<0.001)$. Survival analysis demonstrated a significant association between qSOFA score and time to ICU mortality, with patients who had higher qSOFA scores dying sooner after ICU admission ( $p=0.001$ ). There was a linear increase in probability of in-hospital mortality as qSOFA score increased.

\section{qSOFA parameters and ICU mortality}

\begin{tabular}{lcc} 
Table IV: ICU mortality per qSOFA parameter & \\
\hline $\begin{array}{l}\text { qSOFA parameter } \\
\text { present }\end{array}$ & $\begin{array}{c}\text { ICU mortality when specific } \\
\text { parameter present }\end{array}$ & p-value \\
\hline $\begin{array}{l}\text { Systolic blood pressure } \\
\leq 100 \mathrm{mmHg}\end{array}$ & $56.7 \%$ & $<0.001$ \\
$\begin{array}{l}\text { Altered mentation } \\
(\mathrm{GCS}<15)\end{array}$ & $65.5 \%$ & $<0.001$ \\
$\begin{array}{l}\text { Respiratory rate } \geq 22 \\
\text { breaths per minute }\end{array}$ & $46.0 \%$ & 0.266 \\
\hline
\end{tabular}

Using univariate logistic regression, systolic blood pressure and altered mentation were both independent predictors of ICU mortality $(p<0.001)$. The respiratory rate on its own was not a significant predictor of ICU mortality.

\section{Non-modifiable factors}

\section{Comorbidities and outcomes}

Univariate analysis showed no significant association between singular comorbidities and ICU mortality. Multivariate analysis also showed no significant association between number of comorbidities and ICU mortality after adjusting for qSOFA scores.

\section{Discussion}

The qSOFA score was designed to improve the identification of patients with a poor predicted outcome likely due to sepsis using clinical criteria. ${ }^{3}$ This study analysed whether longer time to ICU admission from a qSOFA score $\geq 2$ was associated with mortality. However, it was found that the duration of a qSOFA score $\geq 2$ prior to ICU admission as a single variable was not an independent predictor of the primary outcomes, namely: ICU mortality, in-hospital mortality and length of ICU stay.

There may be several reasons for these findings. It is possible that multiple variables prior to ICU admission, which were not measured in this study, influenced outcomes. Appropriate care prior to ICU which includes appropriate antibiotic therapy (timing, dosage and therapeutic drug monitoring), fluid resuscitation and source control was not evaluated.14,15 The treatments received prior to ICU admission were not standardised either, as patients had come from various wards or directly from theatre, with various attending physicians responsible for their care.

Among patients with a qSOFA score $\geq 2$, there was a wide time range noted before admission to ICU (range 0 to 2555 hours). The reasons for delay in admission of patients with a qSOFA score $\geq 2$ could be due to limitations in the resources available. Resource limitation is an important factor, due to the limited number of critical care beds, nursing staff and doctors available, which reflects larger provincial and nation-wide shortages. ${ }^{16}$ In 2009 , the ratio of ICU beds to population in KwaZulu-Natal was 1:45 000. ${ }^{16}$ Patients in this study that met a qSOFA score $\geq 2$ in the ward or high care may have not had immediate access to an intensive care unit due to various resource limitations. In these situations, the resuscitation, monitoring, source control and antibiotics are initiated outside the critical care environment and continued until a bed is available in ICU. Another potential reason for a significant time delay to ICU admission is failure to recognise sepsis and septic shock. Adding to the complexity of diagnosing sepsis and septic shock is the similar clinical presentation of such varied pathologies as infection, burns or trauma. ${ }^{17}$ Host immune response and causative organisms are variable and further affect the presentation of infection and sepsis. ${ }^{18}$ This diagnostic challenge led to the development of the qSOFA and SOFA scoring algorithms, as the previously used SIRS (systemic inflammatory response syndrome) criteria lacked specificity. ${ }^{4}$ There have since been several studies to compare the efficacy of these scoring systems in the diagnosis of sepsis. A prospective study by Luo et al. found that qSOFA had a lower sensitivity (53\% vs $98 \%$ ) and higher specificity ( $87 \%$ vs $18 \%$ ) in comparison to SIRS. ${ }^{6}$ The authors suggest that the poor sensitivity could be due 
to sepsis related physiological disturbances not accounted for in the qSOFA criteria, such as hypoxaemia, acute kidney injury, hyperbilirubinaemia and coagulopathy. Additionally, a qSOFA score may be positive in non-sepsis related disease processes such as a pulmonary embolus or cardiac failure.

In another study, Churpek et al. found qSOFA to be a relatively late predictor of deterioration compared to SIRS. For example, most patients met SIRS criteria $(\geq 2)$ up to 12 hours earlier than qSOFA criteria ( $\geq 2$ ) prior to a composite outcome (death or ICU admission). ${ }^{7}$ SIRS, while less specific, may be superior in the early detection and management of infection prior to progression to severe organ dysfunction. ${ }^{19}$ Sartelli et al. have also found that the qSOFA score is a not an adequate screening tool as it lacks sensitivity and does not prioritise the early detection of infection, when treatment may have a larger impact on outcome. ${ }^{20}$ Early diagnosis may, in future, be further augmented by the measurement of novel septic biomarkers. ${ }^{21,22}$

Mortality rates for sepsis in ICU globally is estimated to be $25.8 \%,{ }^{1}$ however we found a mortality rate of $44.3 \%$ in this study population. We found mortality rates in this study to be higher per qSOFA score than previously described in other studies. ${ }^{3}$ Independent non-modifiable factors such as age and comorbidities were considered to be possible contributors to mortality, but were not subsequently found to be significant factors. Components of the qSOFA score, low systolic blood pressure and altered mentation were, however, independent predictors of ICU mortality.

Evidence promotes implementing the Surviving Sepsis Campaign ${ }^{23}$ bundles of care as early appropriate treatment may lead to superior outcomes regardless of early admission to ICU. ${ }^{14}$ Strict application of these measures ${ }^{24}$ in some environments has shown improvement in mortality rates, but even with good adherence, mortality may be as high as $29 \% .{ }^{25}$ These persistently high mortality rates may be due to non-modifiable factors or a failure to meet therapeutic targets, which continue to be defined.

Future research will hopefully lead to expedited identification of pathogens and drug susceptibility testing to make directed treatment more effective, ${ }^{26}$ and genetic testing may assist in predicting the potential for a dysregulated host response to infection. ${ }^{27}$ Therapeutic targets for microvascular dysfunction are also under continual investigation. ${ }^{28}$

More studies are required in low- and middle-income countries, such as South Africa, to allocate resources appropriately and improve management of this often-fatal disease process. This study focused on short term outcomes, but it is important to note that the devastating consequences of sepsis may continue after ICU discharge. ${ }^{29} \mathrm{It}$ is therefore imperative to evaluate the strengths and limitations of qSOFA as a predictive tool in clinical settings. The available research suggests that it may alert clinicians to potential adverse outcomes, but whether these outcomes are ultimately modifiable remains unknown.

\section{Limitations}

This is a retrospective, single centre study.

Data collection was dependent on accurate recordings of vital signs - inaccurate recording of notes may have led to miscalculations of the qSOFA score.

Incorrectly entered admission diagnosis on the ICU database may have affected the study population size. Inclusion criteria limits generalisability to adult patients. Treatment prior to ICU admission was not standardised.

\section{Conclusions}

In adult patients with suspected infection, the duration of a qSOFA score $\geq 2$ prior to ICU admission had no significant association with ICU mortality, in-hospital mortality and length of ICU stay. An increasing qSOFA score was significantly associated with higher ICU mortality.

\section{Conflict of interest}

The authors have no conflict of interest to declare.

\section{Funding source}

None.

\section{Ethics approval}

Approval attained from the Biomedical Research Ethics Committee at the University of KwaZulu-Natal - BREC Reference number BE003/18.

\section{ORCID}

S Pillay (iD https://orcid.org/0000-0001-6643-1688

T Kisten iD https://orcid.org/0000-0002-1609-6962 HM Cassimjee (iD https://orcid.org/0000-0002-2732-1889

\section{References}

1. Vincent JL, Marshall JC, Namendys-Silva SA, et al. Assessment of the worldwide burden of critical illness: the intensive care over nations (ICON) audit. Lancet Respir Med. 2014;2(5):380-6. https://doi.org/10.1016/S2213-2600(14)70061-X.

2. Seymour CW, Liu VX, Iwashyna TJ, et al. Assessment of Clinical Criteria for Sepsis: For the Third International Consensus Definitions for Sepsis and Septic Shock (Sepsis-3). Jama. 2016;315(8):762-74. https://doi.org/10.1001/jama.2016.0288.

3. Freund $Y$, Lemachatti $N$, Krastinova $E$, et al. Prognostic accuracy of Sepsis-3 criteria for in-hospital mortality among patients with suspected infection presenting to the emergency department. Jama. 2017 Jan;317(3):301-8. https:// doi.org/10.1001/jama.2016.20329.

4. Singer M, Deutschman CS, Seymour CW, et al. The Third International consensus definitions for sepsis and septic shock (Sepsis-3). Jama. 2016:315(8):801-10. https://doi.org/10.1001/jama.2016.0287.

5. Raith EP, Udy AA, Bailey M, et al. Prognostic accuracy of the SOFA score, SIRS criteria, and qSOFA score for in-hospital mortality among adults with suspected infection admitted to the intensive care unit. Jama. 2017;317(3):290-300. https:// doi.org/10.1001/jama.2016.20328.

6. Luo J, Jiang W, Weng $L$, et al. Usefulness of qSOFA and SIRS scores for detection of incipient sepsis in general ward patients: A prospective cohort study. J Crit Care Med. 2019;51:13-8. https://doi.org/10.1016/j.jcrc.2019.01.012.

7. Churpek MM, Snyder A, Han X, et al. Quick sepsis-related organ failure assessment, systemic inflammatory response syndrome, and early warning scores for detecting clinical deterioration in infected patients outside the intensive care unit. Am J Respir Crit Care Med. 2017;195(7):906-11. https://doi. org/10.1164/rccm.201604-08540C.

8. Rudd KE, Seymour CW, Aluisio AR, et al. Association of the Quick Sequential (Sepsis-Related) Organ Failure Assessment (qSOFA) score with excess hospital mortality in adults with suspected infection in low- and middle-income countries. Jama. 2018;319(21):2202-11. https://doi.org/10.1001/jama.2018.6229.

9. Huson MA, Katete $C$, Chunda $L$, et al. Application of the qSOFA score to predict mortality in patients with suspected infection in a resource-limited setting in Malawi. Infection. 2017;45:893-896. https://doi.org/10.1007/s15010-017-1057-5.

10. Huson MA, Kalkman R, Grobusch MP, Van der Poll T. Predictive value of the qSOFA score in patients with suspected infection in a resource limited setting 
in Gabon. Travel Med Infect Dis. 2017;15:76-7. https://doi.org/10.1016/j. tmaid.2016.10.014.

11. Adhikari NK, Rubenfeld GD. qSOFA score for patients with sepsis in low- and middle-income countries. Editorial. Jama. 2018;319(21):2175-7. https://doi. org/10.1001/jama.2018.6413.

12. Lundberg JS, Perl TM, Wiblin T, et al. Septic shock: an analysis of outcomes for patients with onset on hospital wards versus intensive care units. Crit Care Med. 1998;26(6):1020-4. https://doi.org/10.1097/00003246-199806000-00019.

13. Shorr A, Choe $Y$, Linde-Zwirble W. Delayed ICU admission with communityacquired severe sepsis greatly increases mortality and resource use. Crit Care. 2011;15(1):P211. https://doi.org/10.1186/cc9631.

14. Gonçalves-Pereira J, Pereira J, Ribeiro 0 , et al. Impact of infection on admission and of the process of care on mortality of patients admitted to the Intensive Care Unit: the INFAUCI study. Clin Microbiol Infect. 2014;20(12):1308-15. https:// doi.org/10.1111/1469-0691.12738.

15. Cardoso T, Carneiro AH, Ribeiro O, Teixeira-Pinto A, Costa-Pereira A. Reducing mortality in severe sepsis with the implementation of a core 6-hour bundle: results from the Portuguese community-acquired sepsis study (SACiUCI study). Crit Care. 2010;14(3):R83. https://doi.org/10.1186/cc9008.

16. Naidoo K, Singh J, Lalloo U. A critical analysis of ICU/HC beds in South Africa: 2008-2009. S Afr Med J. 2013;103(10):751-3. https://doi.org/10.7196/SAMJ.6415.

17. Vincent J-L. The clinical challenge of sepsis identification and monitoring. PLOS Med. 2016;13(5):e1002022-e. https://doi.org/10.1371/journal.pmed.1002022.

18. Angus DC, Van der Poll T. Severe sepsis and septic shock. 2013;369(9):840-51. https://doi.org/10.1056/NEJMra1208623.

19. Simpson SQ. New sepsis criteria: a change we should not make. Chest. 2016;149(5):1117-8. https://doi.org/10.1016/j.chest.2016.02.653.

20. Sartelli M, Kluger $Y$, Ansaloni $L$, et al. Raising concerns about the Sepsis-3 definitions. World J Emerg Surg. 2018;13:6. https://doi.org/10.1186/ s13017-018-0165-6.
21. Kofoed K, Andersen O, Kronborg G, et al. Use of plasma C-reactive protein, procalcitonin, neutrophils, macrophage migration inhibitory factor, soluble urokinase-type plasminogen activator receptor, and soluble triggering receptor expressed on myeloid cells-1 in combination to diagnose infections: a prospective study. Criti Care. 2007;11(2):R38. https://doi.org/10.1186/cc5723.

22. Gibot $S$, Béné $M C$, Noel $R$, et al. Combination biomarkers to diagnose sepsis in the critically ill patient. Am J Respir Crit Care Med. 2012;186(1):65-71. https://doi. org/10.1164/rccm.201201-00370C.

23. Rhodes A, Evans LE, Alhazzani W, et al. Surviving Sepsis Campaign: International guidelines for management of sepsis and septic shock. Intensive Care Med. 2017 Mar;43(3):304-77.

24. Dellinger RP, Carlet JM, Masur $\mathrm{H}$, et al. Surviving Sepsis Campaign guidelines for management of severe sepsis and septic shock. Crit Care Med. 2004;32(3):85873. https://doi.org/10.1097/01.CCM.0000117317.18092.E4.

25. Levy MM, Rhodes A, Phillips GS, et al. Surviving Sepsis Campaign: association between performance metrics and outcomes in a 7.5-year study. Crit Care Med. 2015:43(1):3-12. https://doi.org/10.1097/CCM.0000000000000723.

26. Chun K, Syndergaard C, Damas C, et al. Sepsis pathogen identification. J Lab Autom. 2015;20(5):539-61. https://doi.org/10.1177\%2F2211068214567345.

27. Scerbo MH, Moore L. Early management of sepsis, severe sepsis, and septic shock in the surgical patient. In: Moore L, Todd S, editors. Common problems in acute care surgery. New York: Springer; 2017. p. 71-93. https://doi. org/10.1007/978-3-319-42792-8_7.

28. Penna GL, Salgado DR, Japiassú AM, et al. Microcirculatory assessment: a new weapon in the treatment of sepsis? Rev Bras Ter Intensiva. 2011;23(3):352-7.

29. Winters $B D$, Eberlein $M$, Leung J, et al. Long-term mortality and quality of life in sepsis: a systematic review. Crit Care Med. 2010;38(5):1276-83. 